\title{
oints
}

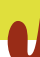

\section{Medial unicondylar knee arthroplasty: technical pearls}

\author{
FILIPPO BONIFORTI
}

Department of Orthopaedics, Foundation San Raffaele Giglio, Cefalù, Italy

\begin{abstract}
Unicondylar knee arthroplasty implantation is extremely demanding as the prosthesis needs to be integrated in the natural anatomy of the knee. It ensures the integrity of the natural knee kinematic. Some studies and registries data have shown lower success rate in comparison with total knee arthroplasty, and patientrelated factors may have an impact on outcome. While, better results have been published by high volume centres. The indications for surgery should be reconsidered critically, even if medial osteoarthritis of the knee remains the most common. This article sets out the diagnostic, and surgical steps in order to fine tuning the unicompartmental replacement of the knee.
\end{abstract}

Key Words: biomechanics, knee, osteoarthritis, replacement, unicompartmental.

\section{Introduction}

Unicondylar knee arthroplasty (UKA) has been promoted for unicompartmental disease of the knee joint. The suggested benefits of UKA over total knee replacement are a shorter rehabilitation time, a greater average range of motion, and preservation of the proprioceptive function of knee ligaments with a more natural-feeling knee (1). While the indications for UKA are debatable, the contraindications are fairly definite: arc of motion less than $90^{\circ}$, angular deformity greater

\footnotetext{
Corresponding Author:

Filippo Boniforti, MD

Department of Orthopaedics, Fondazione San Raffaele Giglio

90015 Cefalù, Italy

E-mail: filippo.boniforti@gmail.com
}

than $15^{\circ}$, and anterior cruciate ligament (ACL) deficiency suggesting the need for a total knee implant (2). Long-term survival results for UKA have been published and registry data are available to describe performances (3). It has been suggested that a bone-sparing operation such as UKA will later allow an uncomplicated revision. However to date, revisions of previous UKAs have not demonstrated this benefit (4).

Surgical technique plays significant role in UKA outcomes and knowledge of the hardware involved is important. The implants on the market differ in prosthesis design, biomechanics, material properties and design aspects. However, we should be guided through all the steps of the operation by certain surgical principles, and the reliability of our performance depends on a proper understanding of these principles prior to surgery. The replaced compartment of the knee has to be matched with the two healthy ones, and, in this regard, performance is linked to surgeon skill and surgeon volume (5). Greater surgeon experience in knee arthroscopy makes for easier intraoperative maneuvering of the knee with all the ligaments, and greater experience in joint replacement makes for easier bone cutting and alignment of components. Possession of these two areas of expertise is a great advantage when performing unicompartmental knee replacements.

\section{Surgical technique}

In the operating room, the patient lies supine on the table, preferably under local epidural anesthesia. The lower limb is draped and maneuverable. A leg holder maintains the $90^{\circ}$ of flexion and valgus stress position. A tourniquet is advocated to provide a bloodless operating field. This may facilitate identification of bony landmarks and prosthesis fixation, even though it may create some pressure on the thigh or reduce quadriceps excursion (6). With the knee bent $90^{\circ}$, make a longitudinal skin incision from the medial bor- 
der of the patella down to the joint line distally (this was originally described as Von Langenbeck's approach). The length of the skin incision depends on different factors (patient, deformity, instruments and surgeon characteristics): 8 to 10 centimeters should be sufficient in most cases, but a less invasive incision (within 6 centimeters) is also possible. The skin flap should be kept as thick as possible by ensuring that the dissection remains just superficial to the extensor mechanism. Inferiorly and superiorly to the medial patella edges, the geniculate vessels are identified and cauterized or ligated. Proceed with the retinacular medial incision, extending 3 to $4 \mathrm{~cm}$ proximally along the quadriceps tendon, leaving a vastus medialis cuff for later closure, and distally onto the anteromedial surface of the tibia. Open the joint space. The knee is extended and the patella dislocated laterally. Continue the dissection to the infrapatellar bursa, being careful to protect the coronary ligament and avoiding detachment of the anterior horn of the lateral meniscus. Inspect the patella femoral joint and the lateral compartment. Bend the knee and remove the medial border of the fat pad; detach the coronary ligament at the anterior horn of the medial meniscus. Ligate or cauterize vessels to keep the knee dry and clean. The infrapatellar branch of the saphenous nerve is sometimes cut with incisions that stray too far medially. Use saline lavage frequently. The medial meniscus is detached from the tibial border and the medial compartment of the knee is opened. The ACL is inspected for function and tropism. When the ACL is healthy, degenerative arthritis usually involves only one compartment. When the ACL is loose, check the other compartments. The possibility of converting to total knee replacement should always be kept in mind (7). The patient must be informed of this possibility and has given his consent before the operation, even in cases in which there were no doubts over the diagnosis. Once unicompartmental involvement has been established, proceed with the removal of peripheral osteophytes, usually on the femoral and tibial borders. This allows correction of varus deformity in most cases. Retractors are helpful in keeping the patella dislocated laterally and in protecting the medial collateral ligament. Bone cuts can start either from the femoral or the tibial side. If the tibia is cut first, an extramedullary tibial alignment method can be used to align the cutting guide on the mechanical axis of the tibia, and to establish the proper slope and thickness of the tibial cut. With a reciprocating saw make a cut on the sagittal plane from the apex of the medial tibial spine, along the tibial plateau, while protecting the medial side of the tibial insertion of the ACL (Fig. 1). Next, the horizontal cut of the tibial plateau has to be perpendicular to the tibial mechanical axis: never overcorrect the anatomical varus deformity. To establish the amount of bone to be resected it is necessary to consider the prosthesis size: do not remove more bone than implant thickness. The level of the joint line must be preserved and evaluated using the tibial trial. It is helpful, for restoration of the joint structure, to measure the thickness of the resected bone. Size the implant on the mediolateral and anteroposterior length. Place the component just inside the medial border to avoid impingement with the medial collateral ligament. On the femoral side, most prostheses are the resurfacing type: the amount of resected bone has to be substituted by metal. Alignment may be intramedullary, but the frontal plane has to match the tibial component orientation as well. With the knee bent $90^{\circ}$, keep the femoral component close to the femoral groove of the condyle to reduce overhang of the prosthesis in extension. Cut the posterior condyle to at least the thickness of the femoral implant. Mechanical and anatomical limb alignment, component orientation and joint line preservation are three key points to address in order to implant a lasting UKA (8). With the trials in place and the knee fully extended, the

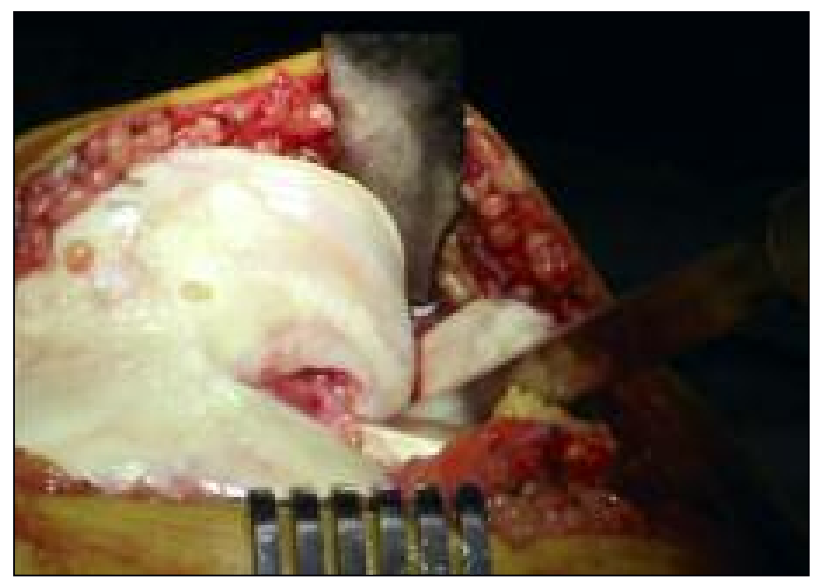

Fig. 1. Bone cuts may start from the femoral or the tibial side. With a reciprocating saw make a cut on the sagittal plane from the apex of the medial tibial spine, along the tibial plateau: protect the medial side of the ACL. 


\section{oints}

F.Boniforti

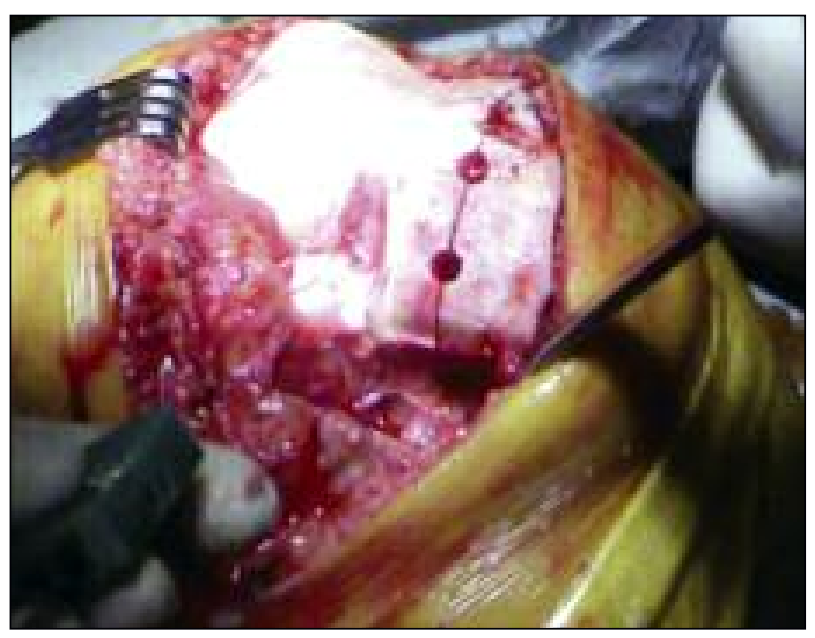

Fig. 2. Knee balance and joint opening under valgus stress have to be assessed in order to over tighten the medial compartment.

medial joint space should be opened one or two millimeters with valgus stress applied. Components placed too tightly can cause the tibia to subluxate toward the opposite compartment and this will produce excessive pressure and wear (Fig. 2). Balance the gaps as for total knee replacement, maneuvering the leg to fine tune the knee tension throughout the arc of movement. Select the insert thickness to achieve proper ligament tension and joint line level.

The cruciate ligaments, which are passive stabilizers of the knee and have a proprioceptive function, make UKR significantly different, biomechanically, from total knee replacement (9). The alignment and balance must be anatomical and symmetrical, after which cementing must be meticulous; any excess cement must be removed (Fig. 3). The postoperative period and rehabilitation program should be very comfortable and straight forward, and most patients are able to return to normal life in four weeks.

\section{Conclusions}

Surgeons today have to be highly skilled in order to obtain the most desirable results from UKA.

The surgical procedure, implant characteristics and technical innovations have to be evaluated taking into

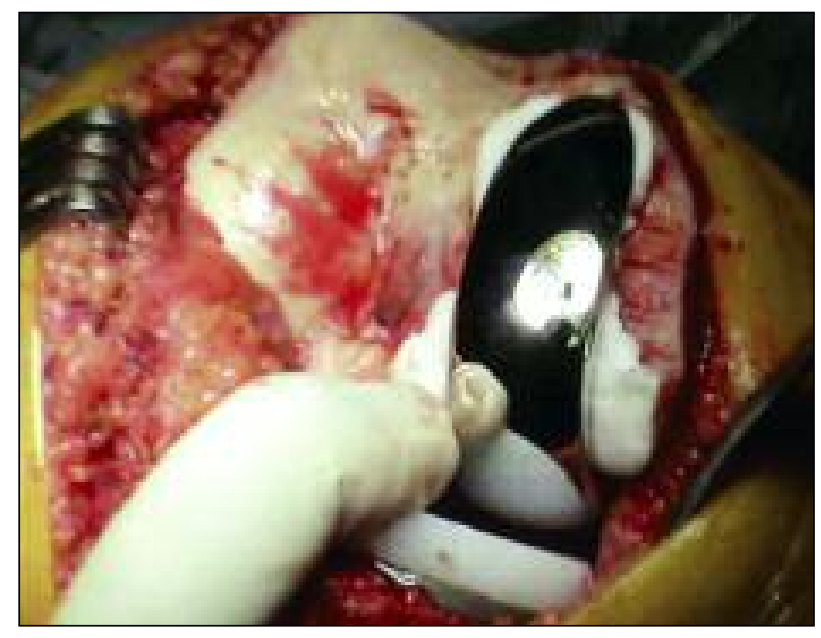

Fig. 3. Mechanical and anatomical limb alignment, component orientation and joint line preservation are three key points to address in order to implant a lasting UKA. Cement in excess has to be removed.

account the patient's expectations, health economics considerations and measurements of quality-adjusted life years.

\section{References}

1. Iorio R, Hearly WL. Unicompartmental arthritis of the knee. J Bone Joint Surg Am. 2003;85-A:1351-1364.

2. Becker R, Argeson JN. Unicondylar knee arthroplasty: what's new? Knee Surg Sports Traumatol Arthrosc. 2013;21:24192420.

3. Phillips JRA, Waterson HA, Searle DJ, Mandalia VI, Toms AD. Registry review: knee arthroplasty. Bone \& Joint. 2014;3:2-8.

4. Robertsson O, W-Dahl A. The risk of revision after TKA is affected by previous HTO of UKA. Clin Orthop Relat Res. 2014;473:90-93.

5. Badawy M, Espehaug B, Indrekvam K, Havelin LI, Fumes O. Higher revision risk for unicompartmental knee arthroplasty in low-volume hospitals. Acta Orthop. 2014;85:342-347.

6. Parvizi J, Diaz-Ledezma C. Total knee replacement with the use of a tourniquet. More pros than cons. Bone Joint J. 2013;95-B(11 Suppl A):133-134.

7. Engh GA, Ammeen DJ. Unicondylar arthroplasty in knees with deficient anterior cruciate ligaments. Clin Orthop Relat Res. 2014;472:73-77.

8. Waterson HB, Philips JRA, Mandalia VI, Toms AD. Thou shalt not varus: still applicable in total knee arthroplasty? Bone \& Joint. 2014;3:9-13.

9. Banks SA, Fregly BJ, Boniforti F, Reinschmidt C, Romagnoli $\mathrm{S}$. Comparing in vivo kinematics of unicondylar and bi- unicondylar knee replacements. Knee Surg Sports Traumatol Arthrosc. 2005;13:551-556. 